\title{
Evaluación del impacto de la acreditación de alta calidad en las instituciones públicas de educación superior de Colombia
}

\author{
Lorenzo Portocarrero-Sierra ${ }^{1}$, Jorge A. Restrepo-Morales ${ }^{2}$ y Jorge E. Arias-Calderón ${ }^{3}$ \\ (1) Tecnológico de Antioquia, Cll 78b 72ª 220, Robledo, Medellín, Colombia (correo-e: loposi@yahoo.es). \\ (2) Facultad de Administración y Ciencias Económicas, Tecnológico de Antioquia, Cll 78b 72ª 220, Robledo, Medellín, \\ Colombia (correo-e: jrestrepo@tdea.edu.co). \\ (3) Facultad de Ciencias de la Administración, Universidad del Valle, Ciudad Universitaria Meléndez Calle 13 \# 100-00, \\ Cali, Colombia (correo-e: jorge.arias@correounivalle.edu.co).
}

* Autor a quien debe ser dirigida la correspondencia

Recibido Feb. 14, 2020; Aceptado Abr. 14, 2020; Versión final Jun. 1, 2020, Publicado Dic. 2020

\begin{abstract}
Resumen
El objetivo de este estudio fue analizar cuáles dimensiones de corte social, económico, financiero y de gestión, diferencian correctamente a las instituciones de educación superior públicas acreditadas de las que no tienen tal reconocimiento. Para ello, se recurre a la técnica de análisis discriminante que evalúa las diferencias significativas y la evolución en los indicadores seleccionados para las 61 instituciones de educación superior públicas que hacen parte del sistema de educación superior en Colombia. El principal hallazgo denota la inequidad en la asignación de recursos a las instituciones de educación superior públicas. Se concluye que las instituciones acreditas, con respecto a las que no tienen esta categoría, perciben el $50 \%$ de los aportes del estado, pero generan una mayor productividad del recurso humano, y por tanto una contribución marginal positiva al desarrollo económico del país.
\end{abstract}

Palabras clave: acreditación; calidad; educación superior; análisis discriminante

\section{Impact evaluation of high quality accreditation in Colombian higher education institutions}

\begin{abstract}
The objective of the present study was to examine what social, economic, financial, and management factors best differentiate accredited from unaccredited higher education institutions in Colombia. Discriminant analysis techniques were used to determine differences and to evaluate selected indicators for 61 public higher education institutions. The main finding denotes the inequity in the allocation of resources to higher education institutions. Accredited institutions receive $50 \%$ of their funding from the state, unlike unaccredited institutions. It is concluded that accredited higher education institutions generate major human resource productivity and have a positive marginal contribution to the country's economic development.
\end{abstract}




\section{INTRODUCCIÓN}

En Colombia, una Institución de Educación Superior (IES), que acredita estándares de calidad debe expresar de forma sistemática y continua la evolución y mejoramiento de los criterios y normas que le soportan tal condición, para ello debe atender de forma razonable con los objetivos, estrategias y planes para cumplir con su objeto social. Sembrar la eficiencia y eficacia de las IES a través de la calidad de sus procesos misionales de investigación, docencia y extensión (Giraldo et al., 2016), como se hace actualmente, producto del enfoque de la nueva gestión pública, lo que exige un proceso técnico de autoevaluación enmarcados en la visión y misión institucional, criterio que infortunadamente no ha sido el factor común en la historia y desarrollo del sistema de educación superior en Colombia en las tres últimas décadas han surgido múltiples centros de educación superior a nivel nacional, como resultado la formación superior sobrelleva transformaciones significativas en las políticas que desembocan en la masificación de la formación, la precariedad académica, deficiente infraestructura y docentes poco calificados lo que redunda en la depreciación de la calidad en el proceso enseñanza-aprendizaje, y como consecuencia una producción de profesionales con serias deficiencias para competir en el mercado laboral (Weber, 2014).

Ante esta situación, existe inquietud en la sociedad, para establecer que las IES practiquen las normas y criterios instaurados para brindar una adecuada educación superior. Con la ley 30 de 1992 se establecieron como órganos rectores al Ministerio de Educación Nacional (MEN) y al Consejo Nacional de Educación Superior (CESU). Dentro del CESU se incluyó la organización del Sistema Nacional de Acreditación, como una estrategia para el mejoramiento de la calidad de la educación superior y del Sistema Nacional de Información, para regular la actividad de acreditación universitaria y a través de éste se fundan y protegen normas de regulación para garantizar a la comunidad en general integridad, calidad y excelencia en las propuestas de valor ofertadas, con estándares que logren la confianza y el respeto del público en general. De acuerdo con el Consejo Nacional de Acreditación (CNA), la Acreditación es el acto por el cual el Estado adopta y hace público el reconocimiento de la institución sobre la calidad de sus programas académicos, su organización y funcionamiento y el cumplimiento de su función social.

La revisión de la literatura arrojo la existencia de un agregado habitual de factores que inciden significativamente sobre la evolución y desarrollo de los sistemas de educación superior, entre los cuales se encuentra el crecimiento y la diversificación, la presencia de la competencia y la dinámica del mercado de educación, las nuevas demandas emanadas de la globalización el advenimiento de la innovación tecnológica y el exponencial cambio de conocimientos (Arechavala et al., 2017). La respuesta a tales demandas, exigen de un sistema de financiación y de gestión avalado por agentes externos, que garanticen la sostenibilidad de los sistemas de educación terciaria. No obstante, en la primera década del presente siglo, el aval externo de calidad, de uso generalizado en el sector primario, fue nuevo para las universidades, entre otras porque tradicionalmente se confiaba en su capacidad de garantizar resultados del aprendizaje aunado a la reactividad y resistencia del sector de la educación para adoptar prácticas de gestión de la calidad (Falabella et al., 2018). Estos factores son aspectos indispensables de las políticas públicas en los países, a partir de la inclusión de mecanismos de revisión robustos (Prisacariu, 2016).

El presente trabajo recurre al análisis discriminante, como técnica de econometría para comprobar hasta qué punto las variables independientes de corte social, económico, financiero y de gestión consideradas diferencian correctamente a las IES acreditadas de las que no tienen tal categoría. En tal sentido, el trabajo plantea como hipótesis central que una IES pública acreditada, financiada con los recursos del Estado, debe demostrar mayor eficiencia en el uso de estos para cumplir con su objeto social.

Para empezar, es importante diferenciar el recurso humano del talento humano, así el recurso humano representa el universo de la organización y por su parte el talento humano se caracteriza por la individualidad y diversidad de profesiones u ocupaciones, a las cuales se refieren las competencias (conocimientos, habilidades, destrezas) y por tanto, la educación pretende fortalecer e identificar el potencial individual, que es considerado un valor único capaz de generar competitividad sostenible (Urrutia-Badillo et al., 2018).

En este trabajo, se considera el concepto de capital humano, bajo la óptica de Becker (2003, pág. 1), quién lo reformula manifestando que se debe entender como "[...] la inversión en dar conocimientos, formación e información a las personas; esta inversión permite a la gente dar un mayor rendimiento y productividad a la economía moderna". Ahora, desde la óptica de la TCH, la educación es definida como una inversión que un individuo efectúa a través del cubrimiento de los costos totales asociados con el proceso de formación con el propósito de incrementar sus competencias (Icarte y Lavate, 2016), sus oportunidades de empleo y sus ingresos futuros, en ese sentido autores como Holden y Biddle (2017) equiparan la posesión de educación como una categoría de capital material susceptible de ser usufructuado y, por tanto, generar una rentabilidad específica. Es así como el concepto de la educación, acorde con los postulados de la TCH, tiene como significado proveer a los trabajadores de "certidumbres" que brinden posibilidades de desarrollo laboral, generando una elevación de los ingresos. 
En ese sentido, diferentes estudios han vinculado educación y crecimiento, como es el caso de Garrido (2007), quien argumenta que en México se han abordado un conjunto de acciones que avocan por la aplicación de una política educativa que, a la luz de los lineamientos de la TCH, favorezca el crecimiento económico; en el mismo país, Cruz et al. (2017) concluyen como los niveles de escolaridad y retornos a la educación responde a un modelo de capital humano. Otro estudio calcula el índice de rentabilidad de la inversión en educación y muestra una mayor rentabilidad de la inversión en educación superior con respecto de cualquier otro nivel de educación inferior. En el caso de Cuba, se corrobora que la contribución de la fuerza de trabajo calificada ha sido positiva y significativa, pero con tendencia a decrecer. Para el caso de España, el capital humano es un factor relevante para el crecimiento económico regional; los coeficientes resultantes de las regresiones corridas resultaron positivos y altamente significativo.

En Chile, sugieren que las ciudades intermedias del sistema urbano chileno requieren políticas regionales de generación, atracción y retención de capital humano para promover una mayor productividad, innovación y desarrollo regional, lo cual tendría efectos en la equidad espacial de ingresos (López-Calva y RodríguezCastelán, 2016). En Malasia, Akhtar, et al. (2017), utilizan un análisis de retornos y beneficios bajo la teoría del capital humano $(\mathrm{TCH})$, para explicar cómo la calidad de los profesores focalizada en formación y entrenamiento afecta las utilidades de las instituciones de educación superior. Para Ecuador, al examinar el efecto del capital humano y la especialización sectorial sobre el valor agregado bruto (VAB) per cápita regional, encontraron evidencia significativa que sugiere una relación directamente proporcional entre el aumento de las competencias del capital humano y la especialización productiva regional con el VAB per cápita regional.

Otro estudio analiza la relación entre la inversión extranjera directa (IED), el crecimiento económico y la desigualdad de ingresos en América Latina, recurriendo a un panel de datos para 18 economías durante el periodo 1980-2009; el hecho destacable es que utilizan el capital humano como variable de control, y en los modelos el coeficiente estimado es negativo y significativo, indicando que a mayor nivel educativo de la población, la desigualdad disminuye, resultado que está en línea con la literatura de capital humano (Murphy y Topel, 2016).

En Colombia, se resalta el papel de la inversión en capital físico y capital humano como motores de crecimiento de los municipios en Colombia; se muestra que los dos son importantes para el desarrollo económico municipal y se concluye la necesidad de fortalecer las políticas dirigidas a mejorar tanto la infraestructura física como una mejor inversión en capital humano, no solo en cantidad, sino en calidad. En correspondencia con esto, se concuerda en afirmar que existe significativa evidencia empírica que apoya la tesis de que, en el modelo de capital humano, la educación es uno de los factores determinantes para mejorar los ingresos; los salarios están estrecha y directamente asociados con la escolaridad, entre más sea ésta, mejores serán las percepciones.

\section{OTROS ANTECEDENTES}

Hay una serie de antecedentes adicionales que es necesario detallar para documentar en mejor forma este trabajo: I) Análisis discriminante; y II) Papel del Estado.

\section{Análisis discriminante}

La técnica del análisis discriminante, ha sido utilizada en gran variedad de estudios para medir la productividad y eficiencia de los sistemas de calidad y otros aspectos de las instituciones educativas, es así como en la India, Kaushalesh, y Shampa (2017), muestran otra interesante aplicación en educación superior al determinar en qué medida el uso de las nuevas tecnologías de información impactan a los estudiantes de educación superior.

Igualmente, Fuller et al. (2016) realizan una exploración empírica de las culturas de evaluación la educación superior en Estados Unidos, que permitió predecir implicaciones para el liderazgo de la evaluación, la práctica y la investigación futura con base en la categorización como pertenecientes a un grupo de aprendizaje o acreditación de estudiantes en función de los puntajes de clasificación lineal. También, Agaoglu (2016) aplica el análisis discriminante para predecir el rendimiento del instructor, el cual muestra que el éxito de los instructores basado en la percepción de los estudiantes depende principalmente del interés de los estudiantes en el curso. Por su parte, Parker (2017) utiliza el análisis discriminante (AD) para determinar los factores de predicción de la deserción escolar en la educación a distancia, ya que estas superan con creces las de la educación tradicional.

En los constructos conceptuales de los elementos que influyen en el rendimiento académico, Tejedor et al. (1991), proponen un índice que sintetiza un grupo complejo de variables de diferente tipo como datos de identificación (sexo, edad, estatus), actitudes cívico-sociales y variables contextuales. Ahora, como resultado 
de esta revisión bibliográfica, se estructura y propone una metodología única para la aplicación del análisis discriminante en la evaluación de la incidencia de la certificación de alta calidad en los indicadores de productividad de las IES públicas en Colombia en los años 2014 y 2016. De igual manera, se determina si existen diferencias significativas y mejoras en los indicadores de productividad seleccionados para estos años.

A partir de 1980, el concepto de capacitación evoluciona hacia la competencia (Salazar, et al., 2018) por lo que las calificaciones obtenidas reflejan un tipo de habilidad apta para enfrentar la "incertidumbre". En este sentido, con la información disponible se construye una variable latente de competencias adquiridas, compuesta por el promedio de los resultados de los estudiantes en las pruebas Saber Pro alcanzadas en inglés, competencias ciudadanas, razonamiento cuantitativo y lectura crítica como proxy del bien material de la educación que habilita a los trabajadores para su desempeño competitivo en el marcado laboral. En ese sentido para el análisis de la distribución de la financiación, con criterios de eficacia, se consideran los inputs del sistema educativo: gasto por estudiante, infraestructura, la relación alumno-maestro y la cobertura como variables claves para medir la eficiencia y eficacia del sistema educativo tanto en el desarrollo de las competencias que garanticen una mayor probabilidad de inserción en el mercado laboral (Brunner, 2018), así como la disminución de las posibles asimetrías en la distribución de los recursos destinados por el Estado para la financiación de las diferentes IES que conforman el sistema de educación superior del país.

\section{Papel del Estado}

A mediados del siglo pasado se produce un cambio conceptual importante que expande las expectativas puestas en el sistema educativo y se impulsa un conjunto de políticas destinadas a elevar las tasas de escolarización propiciando la intervención activa del Estado para garantizar la inclusión igualitaria de la población a la educación, a través de la financiación de ésta. Esta tendencia, contribuyó a la modificación de los patrones del gasto público y de las pautas de justicia redistributiva de la oferta y del financiamiento con criterios de eficacia (Araneda-Guirriman et al. (2018), lo que, en consonancia con las expectativas de movilidad social ascendente, quedó bosquejado un escenario donde predominó crecientemente la demanda de formación. En la actualidad, se recomienda introducir cambios en los criterios y fórmulas de distribución de los recursos del Sistema General de Participación (SGP) para la generación de políticas públicas más equitativas en educación, considerando tanto la eficiencia como la efectividad en la asignación de las transferencias (Villar, 2016). Ante esto, Muñoz (2018) argumenta que las políticas educativas en países de Centroamérica, se han orientado al aumento de gasto en educación, reformas curriculares y reglas de reclutamiento de docentes.

En esta dinámica al Estado le corresponde intervenir las instituciones para que entreguen una educación de calidad con docentes capacitados (Martínez y Romero, 1999) una vez que la exigua efectividad de la docencia es señalada en múltiples estudios como causa del bajo rendimiento escolar en todo el sistema, desde la primaria hasta la formación profesional. En ese sentido, el Estado no puede considerar inversiones universales, sino de desembolsos selectivos, mediante indicadores de validez, que permitan direccionarlos hacia IES que demuestren altos estándares de eficiencia y calidad, con el propósito de potenciar y volver más fructífero el gasto público. Para tales efectos, en Colombia, se define la acreditación como "el reconocimiento por parte del Estado de la calidad de instituciones de educación superior y de programas académicos, es una ocasión para valorar la formación que se imparte con la que se reconoce como deseable con relación a su naturaleza y carácter, y la propia de su área de conocimiento. También es un instrumento para promover y reconocer la dinámica del mejoramiento de la calidad y para precisar metas de desarrollo institucional y de programas" (Consejo Nacional de Acreditación, 2018, pág. 1).

Estudios que pretenden medir esta dinámica, se tiene el de quienes argumentan la importancia de centrar el interés no sólo en cuántos se educan, sino medir el impacto sobre los niveles de productividad de la economía, además argumentan como el mercado laboral prefiere trabajadores bien formados, y delega a los no preparados a las ocupaciones menos productivas y peor remuneradas, con una contribución menor al desarrollo económico.

\section{METODOLOGÍA}

Este trabajo se desarrolla bajo un tipo de investigación explicativa, cuya población son las I.E.S de Colombia, y la muestra alcanza 61 I.E.S que representan el $100 \%$ de las I.E.S. públicas de Colombia. El objetivo fue analizar cómo se diferencian las I.E.S acreditadas de las que no tienen esta categoría, verificando la incidencia en la eficiencia y eficacia en el uso de los recursos. El diseño de la investigación es de corte longitudinal, recurriendo a información de 3 años. Se aborda un enfoque mixto de corte descriptivo propositivo, soportado en un análisis discriminante multivariado (ADM), para evidenciar y contrastar la incidencia de la certificación de alta calidad en la eficiencia y eficacia del sector analizado. Es una técnica que permitió comprobar hasta qué punto las variables independientes de corte social, económico, financiero y de gestión consideradas diferencian correctamente a las IES acreditadas y las que no lo son. 
El modelo de mejor ajuste utilizó como variables explicativas el aporte promedio de la nación por estudiante, los resultados de las pruebas saber pro, los docentes por cada cien estudiantes, la participación de las IES públicas de Colombia en el total de matrículas del sistema (incluyendo las privadas) y el indicador de empleabilidad. La hipótesis central subyace en que las I.E.S acreditadas son más eficientes y eficaces que las demás del sistema y la dimensión de mayor incidencia es la económica. Los resultados se lograron mediante un análisis discriminante multivariado (ADM), debido a que éste permitió identificar los factores, dimensiones o variables, medidas tanto en objetos como en individuos, que explican mejor los atributos que diferencian los grupos a los cuales pertenecen dichos objetos o individuos.

Al-karkhi y Alqaraghuli (2019), expresan que el resultado final del análisis discriminante es un modelo que puede ser utilizado para predecir la membresía a un determinado grupo, comprendiendo la relación entre el conjunto de variables seleccionadas y las observaciones. Para ello, se recurre también al análisis de correspondencias múltiple (MCA), relacionado con el análisis de factores y el escalado multidimensional (MDS). Tanto MCA como MDS son útiles para visualizar datos en forma de mapas perceptuales, generalmente un gráfico de dos o tres dimensiones.

Para la realización de esta investigación se utilizaron los indicadores presentados en la Figura 1. Asimismo, como criterio de selección de las dimensiones y variables que mejor discriminan en la evaluación de la eficiencia y eficacia como resultado de la acreditación de alta calidad en las IES de Colombia, se utilizó la distancia D2 de Mahalanobis, que permite determinar la similitud o diferencia entre dos variables teniendo en cuenta la correlación entre cada una de ellas, lo que permite inferir las variables o indicadores con mayor variación. Toda técnica estadística, exige la comprobación de los supuestos asumidos por el modelo. El análisis discriminante se apoya en los siguientes supuestos: Normalidad multivariante, Igualdad de matrices de varianza-covarianza, Linealidad y Ausencia de multicolinealidad y singularidad.

En primera instancia, se aplica la Técnica del Censo Poblacional en la que todos los elementos son considerados; sin embargo, se excluyen 2 IES que no reportan información en las bases de datos del Sistema Nacional de Información de Educación Superior (SNIES). Se tomaron como fuentes de información, los estados financieros de 2014, 2015 y 2016 (Aporte promedio de la Nación por Estudiante) de cada una de las IESP existentes hasta el 31 de diciembre de 2017; información de las bases de datos del SNIES (Promedio pruebas Saber Pro, Docentes por cada 100 Estudiantes, Participación de la IES en la Matricula) y del observatorio laboral (empleabilidad).

Función Discriminante (X1; X2)
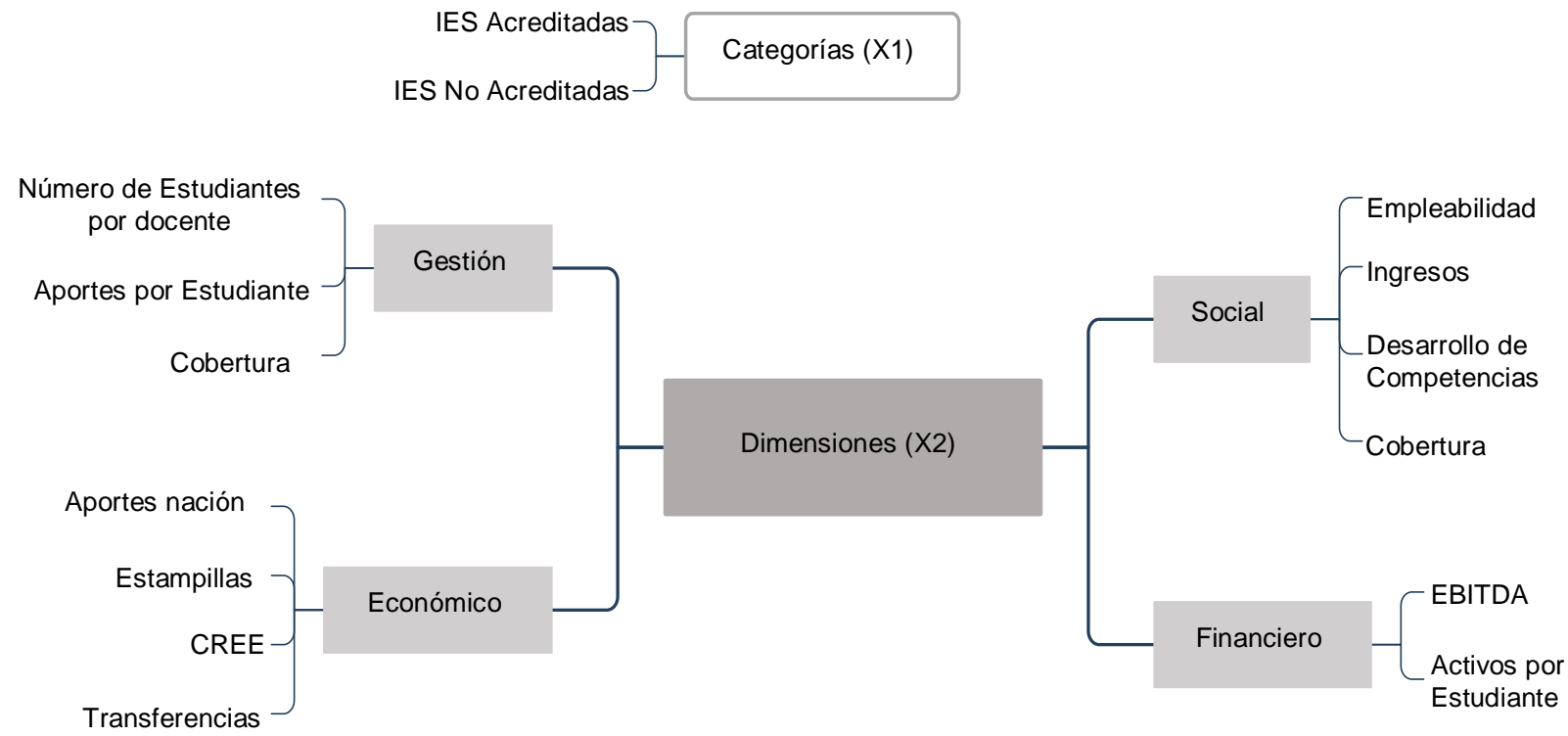

Fig. 1: Categorías, dimensiones e indicadores utilizados. 
Tabla 1: Variables incluidas en el estudio.

\begin{tabular}{|c|c|c|c|}
\hline Dimensión & Variables & Explicación & Fuente \\
\hline \multirow[t]{4}{*}{ Económico } & Aportes Nación & Son los aportes que brinda la nación a cada uno de las IES. & \\
\hline & Estampillas & $\begin{array}{l}\text { Es una fuente de financiación establecida por el gobierno } \\
\text { nacional, que emite estampillas para determinadas I.E.S y esos } \\
\text { recursos financian la operación }\end{array}$ & $\begin{array}{l}\text { Estados financieros } \\
\text { de las IESP }\end{array}$ \\
\hline & Transferencias & $\begin{array}{l}\text { Estos son los aportes que hace la nación a las universidades } \\
\text { estatales u oficiales, en desarrollo de la Constitución y los } \\
\text { artículos } 86 \text { y } 87 \text { de la Ley } 30 \text { de } 1992 .\end{array}$ & $\begin{array}{l}\text { Estados financieros } \\
\text { de las IESP }\end{array}$ \\
\hline & CREE & $\begin{array}{l}\text { EI CREE (Impuesto sobre la Renta para la Equidad) es un } \\
\text { impuesto creado a partir del } 1 \text { de enero de } 2013 \text { a raíz de la } \\
\text { reforma tributaria de } 2012 \text { que llevo a cabo el Congreso de la } \\
\text { República, como aporte de las sociedades y personas jurídicas } \\
\text { y asimiladas en beneficio de los trabajadores, la generación de } \\
\text { empleo y la inversión social. }\end{array}$ & $\begin{array}{l}\text { Decretos de } \\
\text { asignación de } \\
\text { recursos CREE }\end{array}$ \\
\hline \multirow[t]{3}{*}{ Gestión } & $\begin{array}{l}\text { Número de } \\
\text { estudiantes por } \\
\text { docente }\end{array}$ & $\begin{array}{l}\text { Medida de eficiencia de las IES en el uso de los recursos, se } \\
\text { establece una relación entre el número de estudiantes y los } \\
\text { docentes vinculados }\end{array}$ & MIDE \\
\hline & $\begin{array}{l}\text { Aportes por } \\
\text { estudiante }\end{array}$ & $\begin{array}{l}\text { Es la relación entre los aportes recibidos de cada IES dividido } \\
\text { por el número de estudiantes, es una medida de eficiencia }\end{array}$ & $\begin{array}{l}\text { Estados financieros } \\
\text { de las IESP }\end{array}$ \\
\hline & Cobertura & $\begin{array}{l}\text { Es el porcentaje de los estudiantes de cada IES dividido por el } \\
\text { total de estudiantes del sistema }\end{array}$ & SNIES \\
\hline \multirow[t]{4}{*}{ Social } & Empleabilidad & $\begin{array}{l}\text { Mide el nivel de empleabilidad de cada IES, relaciona del total } \\
\text { de egresados que porcentaje se encuentra vinculado al sistema } \\
\text { de seguridad social del país }\end{array}$ & OLE \\
\hline & Ingresos & $\begin{array}{l}\text { Muestra el ingreso promedio del egresado vinculado } \\
\text { laboralmente por cada IES }\end{array}$ & OLE \\
\hline & $\begin{array}{l}\text { Desarrollo de } \\
\text { Competencias }\end{array}$ & $\begin{array}{l}\text { Es el resultado promedio de las pruebas saber pro obtenidas } \\
\text { por cada IES en el ítem de Competencias }\end{array}$ & ICFES \\
\hline & Cobertura & $\begin{array}{l}\text { Es el porcentaje de los estudiantes de cada IES dividido por el } \\
\text { total de estudiantes del sistema }\end{array}$ & SNIES \\
\hline \multirow[t]{2}{*}{ Financiero } & EBITDA & $\begin{array}{l}\text { Utilidades antes de Intereses, depreciaciones, impuestos y } \\
\text { amortizaciones. Es una medida financiera de la situación } \\
\text { financiera de cada IES }\end{array}$ & $\begin{array}{l}\text { Estados financieros } \\
\text { de las IESP }\end{array}$ \\
\hline & $\begin{array}{l}\text { Activos por } \\
\text { estudiante }\end{array}$ & $\begin{array}{l}\text { Relaciona el total de Activos de cada IES sobre el número de } \\
\text { estudiantes. }\end{array}$ & $\begin{array}{l}\text { Estados financieros } \\
\text { de las IESP }\end{array}$ \\
\hline
\end{tabular}

La población de esta investigación está conformada por 61 Instituciones de Educación Superior Públicas de Colombia, con fecha de corte a diciembre de 2017: ocho instituciones técnicas profesionales: Instituto Técnico Nacional de Comercio Simón Rodríguez, Instituto Nacional de Formación Técnica Profesional de San Andrés, Instituto Nacional de Formación Técnica Profesional de San Juan del Cesar, Instituto Tolimense de Formación Técnica Profesional, Colegio Integrado Nacional Oriente de Caldas, Instituto de Educación Técnica Profesional de Roldanillo, Instituto Nacional de Formación Técnica Profesional - Humberto Velásquez García, Instituto Técnico Agrícola; cinco instituciones tecnológicas: Instituto Tecnológico del Putumayo, Instituto Superior de Educación Rural, Unidades Tecnológicas de Santander, Colegio Mayor de Bolívar, Escuela Superior Tecnológica de Artes Débora Arango; 16 instituciones universitarias: Escuela Nacional del Deporte, Escuela Tecnológica Instituto Técnico Central, Colegio Mayor del Cauca, Conservatorio del Tolima, Institución Universitaria Bellas Artes y Ciencias de Bolívar, Instituto Departamental de Bellas Artes, Instituto Tecnológico de Soledad Atlántico, Instituto Universitario de la Paz, Politécnico Colombiano Jaime Isaza Cadavid, Tecnológico de Antioquia, Colegio Mayor de Antioquia, Institución Universitaria Antonio José Camacho, Institución Universitaria de Envigado, Institución Universitaria Pascual Bravo, Instituto Tecnológico Metropolitano, Unidad Central del Valle del Cauca.

A esto se agregan 31 universidades: Universidad de Antioquia, Universidad de Caldas, Universidad de Cartagena, Universidad de Córdoba, Universidad de Cundinamarca, Universidad de La Amazonía, Universidad de La Guajira, Universidad de Los Llanos, Universidad de Nariño, Universidad de Pamplona, Universidad de Sucre, Universidad del Atlántico, Universidad del Cauca, Universidad del Magdalena, Universidad del Pacífico, Universidad del Quindío, Universidad del Tolima, Universidad del Valle, Universidad Distrital - Francisco José de Caldas, Universidad Francisco de Paula Santander, Universidad Industrial de Santander, Universidad Militar Nueva Granada, Universidad Nacional Abierta y a Distancia, Universidad Nacional de Colombia, Universidad Pedagógica Nacional, Universidad Pedagógica y Tecnológica de Colombia, Universidad Popular del Cesar, Universidad Surcolombiana, Universidad Tecnológica de Pereira, Universidad Tecnológica del Chocó Diego Luis Córdoba, Universidad Colegio Mayor de Cundinamarca. 


\section{RESULTADOS Y ANÁLISIS}

Para analizar las variables asociadas a los indicadores seleccionados, se utilizó el análisis discriminante por medio del software SPSS 24, para ello se formularon diferentes funciones discriminantes con sus respectivos estadísticos; esta técnica permitió la estimación en un marco único y analizar si los indicadores evaluados en el mismo contexto presentan diferencias significativas entre IES acreditadas y las que no los son. Después se verificó el cumplimiento de los supuestos requeridos para calcular y establecer la función objetivo y determinar que indicadores discriminaban mejor para evaluar la eficiencia y eficacia como resultado de la certificación. También se calculó la precisión del modelo para predecir el comportamiento futuro de los indicadores en el sector. Por último, con las medias de los indicadores para el sector, se analizó y evaluó el comportamiento y evolución de los diferentes indicadores de productividad de las IES.

\section{Resultados Verificación de los supuestos}

La Tabla 2, muestra como 43 de las 61 IES bajo análisis, fueron válidas para el análisis discriminante. 18 casos no tenían todas las variables discriminatorias definidas en la figura 1. En especial, todas las instituciones técnicas, que no reportaron la información a los respectivos bancos de información como el Sistema Nacional de Información de la Educación Superior (SNIES).

Tabla 2: Casos de análisis válidos.

\begin{tabular}{|l|l|l|l|}
\hline \multicolumn{2}{|l|}{ Resumen de procesamiento de casos de análisis } & \multicolumn{2}{l|}{ Porcentaje } \\
\hline Casos sin ponderar & 43 & 70,5 \\
\hline Válido & Códigos de grupo perdidos o fuera de rango & 43 &, 0 \\
\hline \multirow{4}{*}{ Excluido } & Como mínimo, falta una variable discriminatoria & 0 & 18 \\
\cline { 2 - 5 } & $\begin{array}{l}\text { Faltan ambos códigos de grupo, los perdidos o los que están fuera de rango y, } \\
\text { como mínimo, una variable discriminatoria }\end{array}$ & 0 &, 0 \\
\cline { 2 - 5 } & Total & 18 & 29,5 \\
\hline Total & & 61 & 100,0 \\
\hline
\end{tabular}

De la tabla 3, es posible corroborar la tesis central de que una IES pública acreditada, financiada con los recursos del Estado, demuestra mayor eficiencia en el uso de los recursos para cumplir con su objeto social.

Tabla 3: Media de los grupos.

\begin{tabular}{|l|l|l|l|c|}
\hline \multicolumn{2}{|l|}{ Acreditación de Alta calidad } & Media & $\begin{array}{l}\text { Desviación } \\
\text { Estándar }\end{array}$ & $\begin{array}{l}\text { Casos } \\
\text { Ponderados }\end{array}$ \\
\hline \multirow{3}{*}{$\begin{array}{l}\text { Sin Acreditación de Alta } \\
\text { Calidad }\end{array}$} & Empleabilidad &, 7749 &, 10109 & 29 \\
\cline { 2 - 5 } & Promedio pruebas Saber Pro & 146,0132 & 8,35182 & 29 \\
\cline { 2 - 5 } & Docentes por cada 100 Estudiantes & 7,8225 & 6,15192 & 29 \\
\cline { 2 - 5 } & Participación de la IES en la Matricula & 1,7550 & 1,91752 & 29 \\
\hline \multirow{5}{*}{$\begin{array}{l}\text { Colidad Acreditación de Alta } \\
\text { Cala }\end{array}$} & $\begin{array}{l}\text { Aporte promedio de la Nación por } \\
\text { Estudiante }\end{array}$ & 11,0644 & 26,80131 & 29 \\
\cline { 2 - 5 } & Empleabilidad &, 8121 &, 06049 & 14 \\
\cline { 2 - 5 } & Promedio pruebas Saber Pro & 160,4503 & 9,69131 & 14 \\
\cline { 2 - 5 } & Docentes por cada 100 Estudiantes & 8,9001 & 2,97410 & 14 \\
\cline { 2 - 5 } & Participación de la IES en la Matricula & 3,3373 & 1,67444 & 14 \\
\cline { 2 - 5 } & $\begin{array}{l}\text { Aporte promedio de la Nación por } \\
\text { Estudiante }\end{array}$ & 5,3951 & 3,53632 & 14 \\
\hline \multirow{5}{*}{ Total } & Empleabilidad &, 7870 &, 09087 & 43 \\
\cline { 2 - 5 } & Promedio pruebas Saber Pro & 150,7136 & 11,06477 & 43 \\
\cline { 2 - 5 } & Docentes por cada 100 Estudiantes & 8,1733 & 5,31316 & 43 \\
\cline { 2 - 5 } & Participación de la IES en la Matricula & 2,2701 & 1,97027 & 43 \\
\cline { 2 - 5 } & $\begin{array}{l}\text { Aporte promedio de la Nación por } \\
\text { Estudiante }\end{array}$ & 9,2186 & 22,13527 & 43 \\
\hline
\end{tabular}

La tabla 4, evidencia los resultados obtenidos del estadístico de contraste $M=40,443$ y un valor de $F=12,619$ con una probabilidad asociada $p=0.000$ impide aceptar la hipótesis nula de igualdad de covarianzas de los grupos de discriminación; lo que significa, por un lado, que no existen desviaciones de la normalidad multivariante de alguna variable y por el otro, que la capacidad explicativa de separación de los grupos entre IES con acreditación y sin acreditación, es buena. 
Tabla 4: Test de resultados. prueba M de Box.

\begin{tabular}{|l|l|l|}
\hline \multicolumn{2}{|l|}{ Resultados de prueba } \\
\hline M de Box & Aprox. & 40,443 \\
\hline F & gl1 & 12,619 \\
\cline { 2 - 3 } & gl2 & 3 \\
\cline { 2 - 3 } & Sig. & 14858,957 \\
\hline
\end{tabular}

\section{Determinación del número de funciones discriminantes}

Para determinar el número de funciones discriminantes significativas se llevó a cabo un contraste de hipótesis secuencial. Si denotamos por $k=$ número de funciones discriminantes significativas el proceso comienza con $\mathrm{k}=0$. En el $(\mathrm{k}+1)$-ésimo paso del algoritmo la hipótesis nula a contrastar es:

$$
\mathrm{H}_{\mathrm{o}}: \mathrm{k}+1=\ldots=\min \{\mathrm{G}-1, \mathrm{p}\}=0
$$

Y el estadístico de contraste viene dado por:

$$
\mathrm{T}=\left(n-1-\frac{p+q}{2}\right) \sum_{j=k+1}^{\min \{q-1, p)} \log \left(1+\lambda_{j}\right)
$$

El cual se distribuye como una $\square^{2}(p-k)(q-k-1)$ si $H_{\circ}$ es verdad. El p-valor asociado al contraste viene dado por:

$$
P\left\lfloor\chi_{(p-k)(q-k-1)}^{2} \geq T_{o b s}\right\rfloor
$$

Donde $T_{o b s}$ es el valor observado de T. El contraste para en el primer valor de k para el cual la hipótesis nula $\mathrm{H}_{\mathrm{o}}$ se acepta. Las siguientes tablas permiten determinar el número de funciones discriminantes requeridas. En las Tablas 5 y 6 para los autovalores se utilizó la primera función discriminante canónica en el análisis. se exhibe el Autovalor $=0,782$ y la correlación canónica $=0,663$ obtenidos mediante el programa SPSS 24. Lo que permite inferir que existe una sola función discriminante que de forma significativa $($ sigma $=0,000)$ clasifica las IES en dos grupos de acreditadas y no acreditadas. El valor lambda de Wilks $=0,561$, arroja una segunda conclusión. No obstante que la función discriminante es útil para pronosticar la pertenencia de las IES a los grupos, no todas las variables serán discriminantes. El valor obtenido sugiere cierta similitud entre los grupos $y$, por lo tanto, se requiere estudiar la influencia de cada una de las variables en la función discriminante obtenida. En consecuencia, se realizó la prueba con todas las variables y se eliminaran las no significativas.

Tabla 5: Autovalores.

\begin{tabular}{|l|l|l|l|l|}
\hline \multicolumn{5}{|c|}{ Autovalores } \\
\hline Función & Autovalor & $\%$ de varianza & $\%$ acumulado & Correlación canónica \\
\hline 1 &, $782^{\text {a }}$ & 100,0 & 100,0 &, 663 \\
\hline
\end{tabular}

Tabla 6: Lambda de Wilks.

\begin{tabular}{|l|l|l|l|c|}
\hline \multicolumn{5}{|l|}{ Lambda de Wilks } \\
\hline Prueba de funciones & Lambda de Wilks & Chi-cuadrado & gl & Sig. \\
\hline 1 &, 561 & 23,118 & 2 &, 000 \\
\hline
\end{tabular}

Así mismo, se muestra el resultado obtenido al aplicar la prueba de hipótesis secuencial utilizado para determinar el número de funciones discriminantes significativas en la Tabla 7. En este caso el número máximo de funciones discriminantes posibles es igual a $\min \{2-1,5\}=1$ por lo que sólo será necesario llevar a cabo una prueba de hipótesis.

Tabla 7: Coeficientes de la función discriminante.

\begin{tabular}{|l|l|}
\hline \multicolumn{2}{|c|}{ Coeficientes de función discriminante canónica estandarizados } \\
\hline & Función 1 \\
\hline Aportes de la Nación por Estudiante &,- 482 \\
\hline Puntaje Promedio Pruebas Saber Pro & 1,048 \\
\hline
\end{tabular}

Para determinar que variables discriminan independientemente entre los grupos de IES se estimó la distancia D2 de Mahalanobis y el Lambda Wilks para cada una de ellas, su correspondiente razón $F$ y nivel de significancia para rechazar la hipótesis nula de que las observaciones provienen de la misma población. Se 
consideró un nivel de significación de 0.05 para los estadísticos $\mathrm{F}$ para entrar y $\mathrm{F}$ para eliminar. Para ello se utilizaron los indicadores presentados en la figura 1. Constituyendo las funciones discriminantes, el resultado final del modelo exhibe los coeficientes estandarizados de la función discriminante estimada. La expresión matemática de dicha función vendrá dada por:

$$
D=0.482 Z_{\text {Vaportes de la nación por estudiante }}+1.048 Z_{\text {Vpuntaje promedio pruebas saber pro }}
$$

Donde $Z_{i}$ indica la tipificación de la variable i-ésima. La Tabla 8, muestra como la función discriminante definida ha clasificado correctamente el $90,7 \%$ de las IES, a partir de los aportes de la nación por estudiante y el promedio en las pruebas saber pro. De igual forma se puede inferir que las dos poblaciones estudiadas presentan un error tipo I de $3.4 \%$, un error tipo II de $21.4 \%$, para una efectividad de clasificación total de $90.7 \%$. Las instituciones que reciben mayores aportes de la nación por estudiante y tienen menor éxito en las pruebas saber pro son las que continúan clasificadas sin acreditación.

Tabla 8: Resultados de la clasificación

\begin{tabular}{|c|l|l|l|l|l|}
\hline \multicolumn{2}{|l|}{ Resultados de clasificación } & Acreditación Institucional & Pertenencia a grupos pronosticada & \multirow{2}{*}{ Total } \\
\cline { 3 - 5 } & & Sin Acreditación & Con Acreditación & \\
\hline \multirow{2}{*}{ Original } & \multirow{2}{*}{ Recuento } & Sin Acreditación & 28 & 1 & 29 \\
\cline { 3 - 6 } & & Con Acreditación & 3 & 11 & 14 \\
\cline { 2 - 5 } & \multirow{2}{*}{$\%$} & Sin Acreditación & 96,6 & 3,4 & 100,0 \\
\cline { 3 - 5 } & Con Acreditación & 21,4 & 78,6 & 100,0 \\
\hline
\end{tabular}

Analizando la matriz de estructuras descrita en la Tabla 9, se establecen las correlaciones dentro de grupos combinados entre las variables y las funciones discriminantes canónicas estandarizadas. Variables ordenadas por el tamaño absoluto de la correlación dentro de la función. Se observa que dicha función elimina las variables Empleabilidad clásica y Docentes por cada 100 estudiantes, de la función discriminante. Con las funciones de centroides de grupo, descritas en la tabla 10, las funciones discriminantes canónicas sin estandarizar se han evaluado en medias de grupos, se obtienen las medidas de cada grupo para las funciones, en este caso son diferentes lo que significa que la función discrimina los grupos de IES "Con Acreditación" y "Sin Acreditación". Los valores negativos de las IES clasificadas "Sin Acreditación" ilustran como a mayor valor absoluto de las variables promedio en las pruebas saber pro y aportes de la nación, mayor influencia para que las IES se clasifiquen en el grupo "Con acreditación".

Tabla 9: Matriz de estructuras

\begin{tabular}{|l|l|}
\hline \multicolumn{2}{|l|}{ Matriz de estructuras } \\
\hline & Función 1 \\
\hline Puntaje Promedio Pruebas Saber Pro &, 890 \\
\hline Empleabilidad Clásica &, 531 \\
\hline Aportes de la Nación por Estudiante &,- 138 \\
\hline Docentes por cada 100 Estudiantes &, 117 \\
\hline
\end{tabular}

Los resultados de normalidad para los datos de las IES acreditadas y las que no, asociados a la prueba de Shapiro y Wilk que requiere que los estadísticos estén por encima de 0.8. Las pruebas de normalidad para las variables por separado de las dos categorías de IES dan como resultado que se comportan como variables normales. Sin embargo, Lachenbruch (1975) demostró como el análisis discriminante es poco sensible a las transgresiones de menor importancia de la hipótesis de normalidad. Tabachnick y Fidell (2001) ,precisan sobre la robustez del análisis discriminante frente al tamaño de las muestras, aconsejan un tamaño de muestra superior a 20 datos para grupos similares, lo que brinda solidez al modelo en torno a la violación del supuesto de multinormalidad. En este caso, se utilizaron 61 IES, con un grupo de 19 y otro de 42, por lo que se cumple con la condición requerida.

Tabla 10: Funciones de centroide de grupo

\begin{tabular}{|l|l|}
\hline \multicolumn{2}{|l|}{ Funciones en centroides de grupo } \\
\hline Acreditación Institucional & Función 1 \\
\hline Sin Acreditación &,- 600 \\
\hline Con Acreditación & 1,243 \\
\hline
\end{tabular}




\section{Homogeneidad de matrices de varianza-covarianza}

El supuesto de igualdad de matrices de varianza-covarianza para las categorías de IES se comprobó con la prueba de Box. Esta prueba identifica si las correlaciones en la población de la que se toma la muestra son 0 , lo que significa que cualquier correlación observada en los datos son resultado de la aleatoriedad del proceso de muestreo. Para ello se definen las siguientes hipótesis: i) H_0: Los datos se distribuyen de forma independiente; y ii) H_a: Los datos no se distribuyen de forma independiente. La estadística de prueba es:

$$
Q=n(n+2) \sum_{K=1}^{h} \frac{P k^{2}}{n-k}
$$

donde $n$ es el tamaño de la muestra, $\mathrm{P} \_k$ es la auto correlación de la muestra en el retraso $\mathrm{k}$ y h es el número de retardos que se están probando. Por nivel de significación a, la región crítica para el rechazo de la hipótesis de aleatoriedad es:

$$
Q>\chi_{1-\alpha, h}^{2}
$$

donde $x \_(1-\alpha, h)^{\wedge} 2$ es la $\alpha$ - cuantil de la distribución chi-cuadrado con $\mathrm{h}$ grados de libertad. De acuerdo con la Tabla 11, es posible concluir que los datos se distribuyen de forma independiente

Tabla 11. Resultados de prueba box

\begin{tabular}{|l|l|l|}
\hline \multicolumn{2}{|l|}{ M de Box } & 73,271 \\
\hline $\mathrm{F}$ & $\begin{array}{l}\text { Aprox } \\
.\end{array}$ & 4,092 \\
\cline { 2 - 3 } & gl1 & 15 \\
\cline { 2 - 3 } & gl2 & 2752,078 \\
\cline { 2 - 3 } & Sig. &, 000 \\
\hline
\end{tabular}

\section{Linealidad y multicolinealidad y singularidad}

Los supuestos de linealidad y multicolinealidad y singularidad no serán evaluados debido a que en la formulación del modelo de análisis discriminante se recurrió al método paso a paso; ahora, considerando el criterio de tolerancia para la inclusión de las variables seleccionadas, aquellas variables con una correlación múltiple alta con las demás variables generarán una baja tolerancia y no serían incorporadas en la formulación de la función discriminante. Por último, se recurre al análisis de correspondencias (en realidad, análisis de correspondencia múltiple, MCA para abreviar) el cual está relacionado con el análisis de factores y también es similar al escalado multidimensional (MDS). Tanto MCA como MDS se pueden usar para visualizar datos en forma de mapas perceptuales, generalmente un gráfico en dos dimensiones, pero a veces en tres dimensiones (Vanegas et al., 2018). El análisis de correspondencia funciona con datos categóricos, en este sentido y con la categorización de las instituciones de educación superior en acreditadas y sin acreditación, se procede a construir un mapa perceptual, en la Figura 2, para comparar el promedio de puntaje obtenido en las pruebas saber pro versus el promedio de aportes de la nación por estudiante. Se corrobora la inequidad del sistema de financiación colombiano, una vez que a mayor aporte por estudiante menor resultado promedio en las pruebas saber pro.

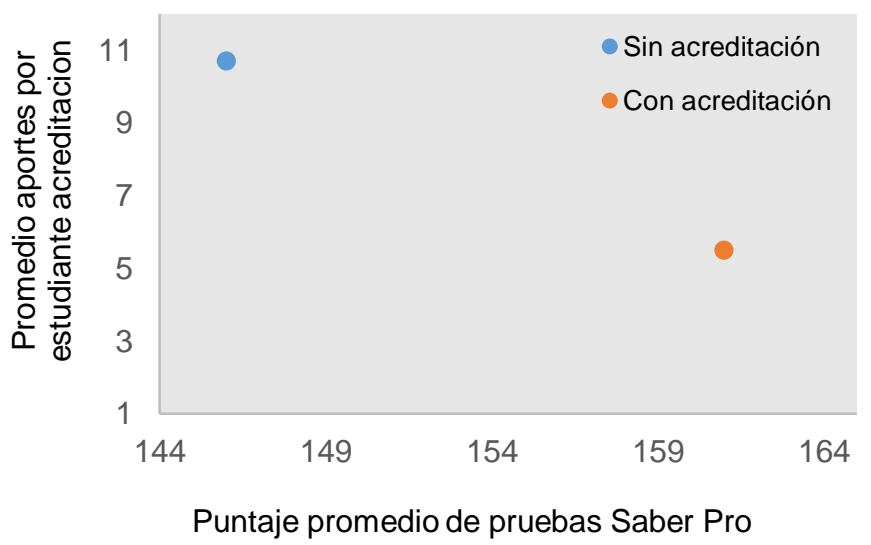

Fig. 2: Mapa perceptual del puntaje promedio en pruebas saber pro y aportes por estudiante de la nación 


\section{DISCUSIÓN}

Al analizar las funciones discriminantes generadas se puede concluir como los indicadores pertenecientes a la dimensión gestión con el indicador aportes por estudiante y la dimensión social con el indicador desarrollo de competencias, son los que mayor impacto tienen en la acreditación. Frente al desarrollo de competencias, en particular los resultados de las pruebas saber pro que tienen una alta correlación con la inversión en investigación, esto confirma la postura de Adrogué et al. (2019) en la que la inversión en los procesos misionales de las instituciones, como es el caso de la investigación, termina por influir en la calidad de los profesionales. En este contexto las universidades en Colombia, como las de los países en vía de desarrollo deben lidiar con recursos escasos como lo confirman Araneda-Guirriman et al. (2018); Sin embargo, para el caso de las dimensiones financiera y económica, los indicadores disponibles no evidencian el mismo comportamiento. Por su parte, la paradoja que se presenta en el sentido de que a menores aportes del Estado por estudiante más probabilidad existe de lograr la acreditación, apunta a enunciar diferentes hipótesis frente a la inequidad en la distribución de los recursos, que van desde la incidencia de la corrupción, pasando por la inequidad en la distribución de los fondos y desembocando en la ineficiente gestión de las IES y falta de voluntad política para lograr la acreditación institucional.

La función discriminante (D) calculada, muestra una relación inversamente proporcional entre los aportes de la nación por estudiante y la acreditación, donde por cada unidad adicional de aportes de la nación se reduce el valor discriminante de clasificación como IES acreditada en 0,482 unidades; en tanto que existe una relación directamente proporcional entre los resultados de las pruebas saber pro y la acreditación, que expresa como por cada unidad que se incremente el promedio de puntaje en las pruebas saber pro, el valor de la predicción de la función discriminante se incrementa en 1,048 unidades. El resultado, aparentemente contradictorio en realidad es una paradoja, porque al revisar la Tabla 2, de las medias de las variables por grupos, se observa como las IES sin acreditación reciben 11,06 unidades por estudiante, mientras que las acreditadas reciben tan sólo 5,39 unidades por estudiante, en una relación de 2,051; lo que permite inferir que a menos recursos de la nación por estudiante mayor es la probabilidad de la IES de pertenecer al grupo de las acreditadas. Esto corrobora lo expuesto por Straubhaar (2016) quien afirma que, en América Latina, en lugar de suscitar la equidad, se ha dado una orientación cada vez mayor en la rendición de cuentas en la política educativa lo que ha dado lugar a un cambio en las prioridades a la evaluación de esos sistemas, en medio de las diferencias generadas.

Lo anterior, paradójico y aparentemente contradictorio, permite plantear una nueva hipótesis sobre fallos en la gestión y distribución de los recursos lo que sugiere la crisis del sistema educativo colombiano, donde una de las causas se podría atribuir a la inequidad del Estado en la distribución de los recursos, situación que requiere una adecuación a las políticas públicas en función de la calidad (Adrogué et al., 2019) y, subyacente sería pertinente emprender estudios encaminados a probar la hipótesis de baja eficiencia en la gestión de las instituciones sin acreditación para administrar los recursos percibidos de la nación como lo afirma Weber (2014). En este sentido, Villar (2016) recomienda introducir cambios en los criterios y fórmulas de distribución de los recursos del Sistema General de Participación para educación que consideren tanto la eficiencia como la efectividad en la asignación de las transferencias. Lo que, sumado a la confirmación de que la formación de capital humano presenta un efecto reductor de la desigualdad en 18 economías de América Latina es un resultado sumamente importante, primero porque se identifica un arma efectiva de lucha contra la desigualdad, y segundo muestra el camino para diseñar políticas públicas en torno a la equidad.

A pesar de que existe evidencia en la literatura de que la medición del rendimiento basada en un conjunto de indicadores constituye una técnica para evaluar la eficiencia de una organización; este trabajo constituye un referente para desarrollar otras investigaciones que evalúen no sólo variables e indicadores asociados con la productividad, sino otras variables internas y externas en diferentes sectores que permitan medir eficacia y competitividad; como es el caso de la gestión del conocimiento, que de acuerdo con Arechava y Sánchez (2017) está lejos de ser adecuada para el desarrollo científico, económico y social de los países, por las divergencias entre instituciones.

\section{CONCLUSIONES}

De acuerdo con el trabajo presentado y los resultados obtenidos, se pueden plantear las siguientes conclusiones principales:

1. El análisis del rendimiento organizacional con base en indicadores admite medir la eficiencia de una organización (Matilla y Chalmeta, 2007); en esta línea el análisis discriminante aplicado logro identificar los indicadores que explican hasta qué punto las variables de corte social, económico, financiero y de gestión consideradas diferencian correctamente a las IES acreditadas y las que no lo son, sobre un grupo de 61 IES públicas de Colombia. 
2. El modelo logro clasificar la población estudiada en dos grupos, evidenciando que existen diferencias significativas en las medias por grupos de algunos indicadores, como el caso de la empleabilidad, el promedio de pruebas Saber Pro, el número de docentes por cada 100 estudiantes, la participación de la IES en la matricula total de estudiantes donde las IES acreditadas aventajan a las no acreditadas.

3. Es paradójico y llama la atención que, en el actual sistema de financiación, el aporte por estudiante de la nación muestre como el rubro asignado a las IES no acreditadas sea el doble de las que sí lo son. No obstante, que las IES acreditadas, reciben la mitad de los recursos por estudiante que las no acreditadas, logran superar con creces todos los indicadores evaluados, en especial el indicador de las pruebas Saber Pro que se comporta como proxi de la incorporación de las competencias que habilitan al profesional egresado de la IES acreditadas, para demostrar un desempeño superior en el mercado laboral.

4. Otro hallazgo importante, permite inferir que no existe evidencia estadística de la incidencia de las dimensiones económica y financiera en los procesos de acreditación de alta calidad. Dado que la gestión del conocimiento es una herramienta que ayudará a ser más competitivos en un entorno cada vez más inestable y cambiante.

5. De igual forma, la investigación permitió establecer una función objetivo para las IES públicas de Colombia, con lo que se puede estudiar y analizar qué indicadores discriminan mejor y, se pueden así, tomar acciones teniendo en cuenta el cálculo y estudio de los indicadores que presentaron una buena discriminación.

\section{REFERENCIAS}

Adrogué, C., García, A., Pita, M., y Salto, D. Las Universidades Frente al Aseguramiento de la Calidad y las Políticas de Financiamiento de la Investigación: Estudios de Caso en el Sector Privado Argentino, doi: 10.36857/resu.2019.190.711, Revista de la educación superior, 48(190), 45-70. (2019).

Agaoglu, M., Predicting Instructor Performance Using Data Mining Techniques In Higher Education, doi: 10.1109/ACCESS.2016.2568756, IEEE Access, 4, 2379-2387 (2016).

Akhtar, S., Awan, S., Ismail, K. y Naveed, S., Social Capital And Learning Organisation: Is It Worth To Engage In Networking?, doi: 10.1504/IJLC.2017.086853, International Journal Of Learning And Change, 9(3), 208-227 (2017).

Al-Karkhi, A. y Alqaraghuli, W., Applied Statistics for Environmental Science With R.1 ${ }^{\text {a }}$ edición, Elseiver, Amsterdam, Netherlands (2019).

Araneda-Guirriman, C., Gairín-Sallán, J., y Pedraja-Rejas, L. La Autonomía en la Educación Superior: Reflexiones desde los Actores en el Contexto del Financiamiento por Desempeño en Chile, doi: 10.4067/S0718-50062018000400065, Formación universitaria, 11(4), 65-74. (2018).

Arechavala, R. y Sánchez, C. Las Universidades Públicas Mexicanas: Los Retos de las Transformaciones Institucionales hacia la Investigación y la Transferencia de Conocimiento, doi: 10.1016/j.resu.2017.09.001, Revista de la educación superior, 46(184), 21-37. (2017).

Becker, G., Capital Humano en la Nueva Sociedad, Presentación en la Fundación DMR (2003).

Brunner. J., Educación Superior y Mundo del Trabajo: Horizontes de Indagación. Calidad En La Educación, doi:10.31619/caledu.n29.195, (29) 230-240 (2018).

Consejo Nacional de Acreditación. Lineamientos para la Acreditación Institucional. Obtenido De Consejo Nacional De Acreditación. ( 2015).

Cruz, A., Díaz, A. y Reyes, R., Un Estudio Empírico Entre la Relación del Capital Humano y el Crecimiento Económico en México. Revista De La Facultad De Contaduría Y Ciencias Administrativas, ISSN: 2448-6051, 1(1), 59-66 (2017).

Falabella, A., Cortázar, A., Godoy, F., González, M. P. y otro autor, Sistemas de Aseguramiento de la Calidad en Educación Inicial Lecciones desde la Experiencia Internacional. Gestión y política pública, ISSN: 14051079, 27(2), 309340. (2018).

Fuller, M., Skidmore, S., Bustamante, R. y Holzweiss, P., Empirically Exploring Higher Education Cultures of Assessment. The Review Of Higher Education, ISSN: 0162-5748, 39(3), 395-429 (2016).

Garrido, C., La Educación Desde la Teoría del Capital Humano y el Otro. Educere, ISSN: 1316-4910, 11(36), 73-80 (2007).

Giraldo, J., Ovalle, D. y Santoro, F. Mecanismo de Inferencia Sensible al Contexto para la Integración de Procesos de Negocio en Acreditación Académica, doi: 10.4067/S0718-50062016000400007, Formación universitaria, 9(4), 53-62. (2016).

Holden, L. y Biddle, J., The Introduction Of Human Capital Theory Into Education Policy in the United States. History Of Political Economy, doi: 10.1215/00182702-4296305, 49(4), 537-574 (2017).

Icarte, A. y Lavate, A. Metodología para la Revisión y Actualización de un Diseño Curricular de una Carrera Universitaria Incorporando Conceptos de Aprendizaje Basado en Competencias, doi: 0.4067/S0718-50062016000200002, Formación universitaria, 9(2), 03-16. (2016). 
Kaushalesh, L. y Shampa, P., New Educational Technologies In Tertiary Education In India: Adoption And Consequences. Journal Of Applied Research In Higher Education, ISSN: 2050-7003, 10(1), 2-14, https://doi.org/10.1108/jarhe-02-20170013 (2018).

Lachenbruch, P. A., Zero-Mean Difference Discrimination And The Absolute Linear Discriminant Function. Biometrika, doi: 10.1093/biomet/62.2.397, 62(2), 397-401, (1975).

López-Calva, L. y Rodríguez-Castelán, C., Pro-Growth Equity: A Policy Framework for the Twin Goals, The World Bank, Washington DC, EE UU (2016).

Martínez, M. y Romero, M., Calidad de la Educación e Integración de las Nuevas Tecnologías. Comunicar: Revista Científica Iberoamericana De Comunicación Y Educación, ISSN: 1134-3478, (13), 183-187 (1999).

Muñoz, J., Politics in Education Quality Policies in Central America, doi: 10.14201/alh201777115136, 77(1). 115-136, (2018).

Murphy, K. y Topel, R., Human Capital Investment, Inequality, And Economic Growth. Journal Of Labor Economics, doi: 10.1086/683779, 34(S2), S99-S127 (2016).

Prisacariu, A. New Perspectives Of Quality Assurance In European Higher Education. Procedia-Social and Behavioral Sciences, doi:10.1016/j.sbspro.2015.02.094, 180(119-126), 660-664. (2015).

Salazar, L., Carrasco, T., Correa, W., Carrillo, J. y otro autor., La Gestión por Competencias una Herramienta Clave para el Desarrollo en el Sector Empresarial, Ciencia Digital, doi: 10.33262/cienciadigital.v2i1.2, 18-30 (2018).

Tabachnick, B. y Fidell, L., Using Multivariate Statistics, $7^{\text {a }}$ Edición. Pearson, Northridge, California (2001).

Tejedor, F., Sabucedo, J., Sobral, J. y Serrano, G., Factores Personales y Contextuales: Su Influencia Sobre el Rendimiento Académico, 1a edición. Tórculo ediciones, Santiago de Compostela, España (1991).

Urrutia-Badillo, Y., Lopez-Cabrales, A. y Valle-Cabrera, R., A Multidimensional Approach to the Content of Human Capital: Dimensions, Emergence Process and Organizational Capabilities, Management Research, Journal Of The Iberoamerican Academy Of Managemen, ISSN: 1536-5433, 138-158 (2018).

Vanegas, J., Restrepo-Morales, J., Barros, G. y Moreno, G., Service Quality in Medellin Hotels Using Perceptual Maps. Cuadernos De Administración, doi: 10.25100/cdea.v34i60.5927, 34(60), 30-45 (2018).

Villar, L., Sistema General de Participaciones y su Vínculo con ILas Políticas e Incentivos Dirigidos a la Excelencia Docente y el Mejoramiento Continuo de la Calidad. Fedesarrollo, Bogotá DC, Colombia (2016).

Weber, S., Human Capital Depreciation and Education Level. International Journal Of Manpower, doi: 10.1108/IJM-052014-0122, 35(5), 613-642. (2014). 
